\title{
An 87-year-old patient with repeated oligorecurrences over six years whose disease were treated with radiotherapy alone
}

\author{
Hyong Geun Yun, MD, PhD \\ Department of Radiation Oncology, Dongguk University Ilsan Hospital, Goyang, Korea
}

\begin{abstract}
In the clinical state of oligometastases or oligorecurrence, a transitional state between localized and widespread systemic disease, local control of the disease may yield improved systemic control. Radiotherapy may be a good means for controlling oligometastatic tumors, particularly in very old patients for whom surgery may be infeasible. A combination of systemic therapy and local therapy is necessary to prevent systemic progression. Some kinds of cancers found in the elderly are known to be somewhat indolent for systemic progression. So, for very old patients who refuse or cannot tolerate chemotherapy, the use of radical radiotherapy alone to treat oligorecurrences may be very helpful. We successfully treated an 87-year-old patient who had been diagnosed with oligorecurrences three times over six years with radiotherapy alone. The patient is now, about four years after his first radiotherapy for liver metastasis, alive without any evidence of cancer and with fully active performance status.
\end{abstract}

Keywords: Oligorecurrence, Oligometastases, Radiotherapy

\section{Introduction}

In the past, cancer metastasis was considered to be the last stage of life, as just one site of metastasis signaled hematogenous seeding, making locoregional therapy useless. About twenty years ago, the clinical state of oligometastatic disease was proposed by Hellman and Weichselbaum [1]. They hypothesized that, in some patients with a limited number of clinically detectable metastatic tumors, the extent of the disease exists in a transitional state between localized and widespread systemic disease. In this model, oligometastatic disease has the potential of progressing to a widespread metastatic disease, and thus, local control of oligometastases may yield improved systemic control. So, in oligometastases, local therapies, such as surgery and radiotherapy, have been suggested to improve the prognosis. Radiotherapy may be a good means to control oligometastatic tumors, particularly in patients who cannot tolerate or do not want surgery, or when tumors are situated in areas in which resection would result in an unacceptable morbidity risk. Some authors [2-6] defined oligorecurrence as oligometastases within a controlled primary site. Of course, a combination of systemic therapy and local therapy is necessary in oligorecurrence to prevent systemic progression. But, very old patients may have an increased susceptibility to toxicity during chemotherapy and may be more reluctant to undergo chemotherapy than their younger counterparts [7]. On the other hand, older patients can tolerate local radiotherapy. In addition, some kinds of cancers in the elderly are known to be somewhat indolent [8-10]. Considering these facts, very old patients with oligorecurrences who do not

Received 14 August 2014, Revised 12 September 2014, Accepted 30 September 2014.

Correspondence: Hyong Geun Yun, MD, PhD, Department of Radiation Oncology, Dongguk University Ilsan Hospital, 27 Dongguk-ro, Ilsandong-gu, Goyang 410-773, Korea. Tel: +82-31-961-7540, Fax: +82-31-961-7564, E-mail: yunhg@dumc.or.kr

(c) This is an Open Access article distributed under the terms of the Creative Commons Attribution Non-Commercial License (http://creativecommons.org/ licenses/by-nc/3.0/) which permits unrestricted non-commercial use, distribution, and reproduction in any medium, provided the original work is properly cited.

www.e-roj.org 
receive systemic chemotherapy for various reasons may still benefit from radical radiotherapy.

\section{Case Report}

An 87-year-old man was admitted for radiation therapy to treat a liver metastasis from colon cancer in September 2010. At admission, he presented with discomfort in the right upper quadrant of his abdomen. His admission laboratory findings showed a total bilirubin of $0.8 \mathrm{mg} / \mathrm{dL}$ (normal, 0.2 to $1.3 \mathrm{mg} /$ $\mathrm{dL}$ ) and direct bilirubin of $0.3 \mathrm{mg} / \mathrm{dL}$ (normal, 0 to $0.3 \mathrm{mg} / \mathrm{dL}$ ). Serum aspartate aminotransferase was $63 \mathrm{IU} / \mathrm{L}$ (normal, 0 to $37 \mathrm{IU} / \mathrm{L}$ ), alanine aminotransferase was $48 \mathrm{IU} / \mathrm{L}$ (normal, 0 to $41 \mathrm{IU} / \mathrm{L}$ ), lactic acid dehydrogenase was 1,152 IU/L (normal, 116 to $243 \mathrm{IU} / \mathrm{L}$ ), alkaline phosphatase was $278 \mathrm{IU} / \mathrm{L}$ (normal, 35 to $129 \mathrm{IU} / \mathrm{L}$ ), and GGT was $282 \mathrm{IU} / \mathrm{L}$ (normal, 8 to $61 \mathrm{IU} /$ L). His carcinoembryonic antigen (CEA) level was markedly elevated to $1,736 \mathrm{ng} / \mathrm{mL}$ (normal 0 to $4.3 \mathrm{ng} / \mathrm{mL}$ ). A whole body ${ }^{18}$ F-fluorodeoxyglucose (FDG) positron emission tomography- computed tomography (PET-CT) revealed a hypermetabolic lesion in the right lobe of the liver (Fig. 1A). The maxSUV (standardized uptake values) was 12.3. Dynamic liver CT showed $10-\mathrm{cm}$ size ill-defined inhomogeneous and partiallycalcified mass in right inferior lobe of liver (Fig. 1B, 1C). And the volume of the mass calculated by the radiotherapy planning computer was $256 \mathrm{~mL}$. The entire liver volume was 1,292 mL.

Previously, the patient underwent low anterior resection for his sigmoid colon cancer in February 2007 in a different hospital. His pathological diagnosis was adenocarcinoma and its pathologic stage was T3NOMO. After the operation, his CEA level was normalized to $3.4 \mathrm{ng} / \mathrm{mL}$. In September 2008 (about six years ago), his CEA level increased to $8.2 \mathrm{ng} / \mathrm{mL}$ from the $3.4 \mathrm{ng} / \mathrm{mL}$ postoperative level. Liver magnetic resonance image showed a $1.3 \mathrm{~cm}$-sized metastatic mass in his 55 liver segment. Metastasectomy was recommended, but he refused and did not receive any anticancer treatments, except some herbal medications.
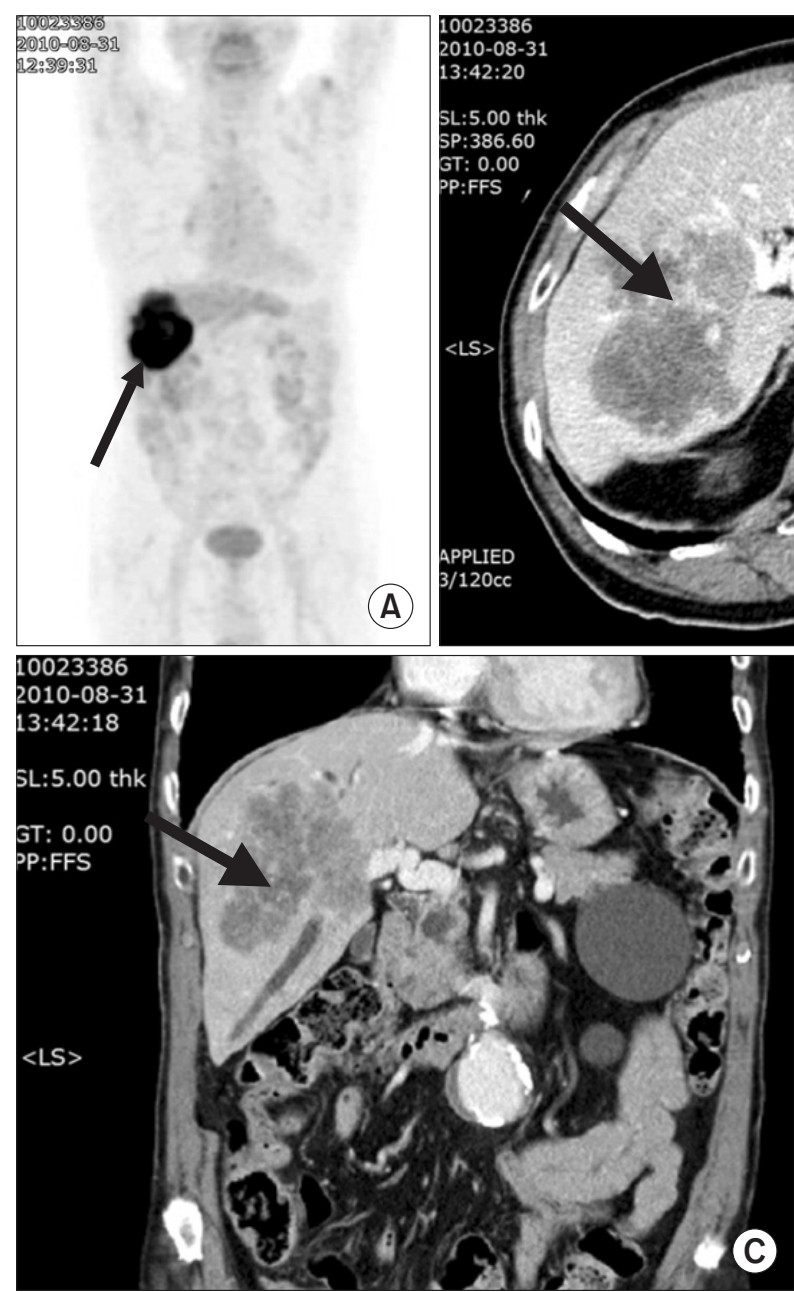

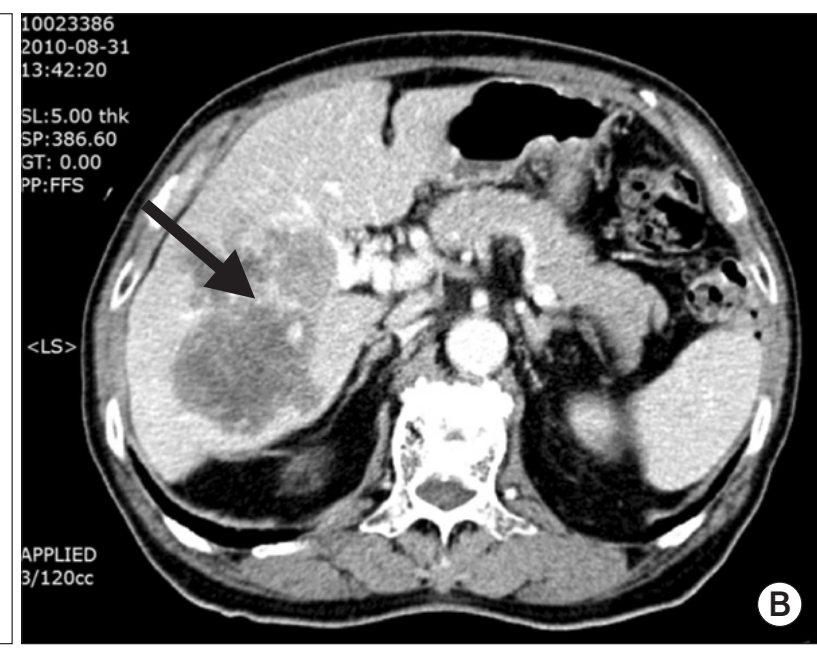

B

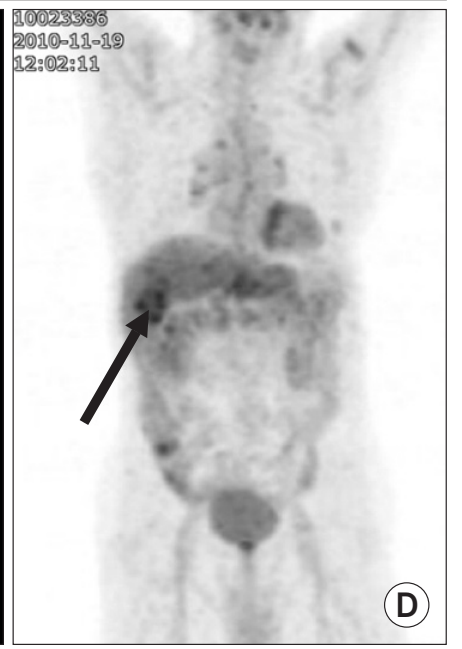

Fig. 1. The liver metastasis regressed rapidly during liver radiotherapy. Arrows denote liver metastasis. (A) In pre-radiotherapy ${ }^{18} \mathrm{~F}$-fluorodeoxyglucose (FDG) positron emission tomography/computed tomography (PET/CT) scan, huge hypermetabolic lesion was observed in liver. (B) Axial and $(C)$ coronal view image of dynamic liver CT scan before liver radiotherapy showed $10-\mathrm{cm}$ size illdefined inhomogeneous and partiallycalcified mass in right inferior lobe of liver. CT scan also showed occlusions of posterior branch and compression of anterior branch of right portal vein was also observed on CT scan. (D) FDG PET/CT scan at the latter part of radiotherapy showed the hypermetabolic lesion in liver was markedly decreased. 


\section{Hyong Geun Yun}

After the development of liver metastasis symptoms, the patient was admitted to our hospital. At first, we prescribed oral capecitabine (Xeloda) with radiation treatment for concurrent chemoradiation, but he stopped chemotherapy due to nausea and abdominal cramping after only a few days of treatment. Consequently, radiotherapy alone was performed for his liver metastasis from October 13, 2010 to December 2,2010 . The total radiation dose was $60 \mathrm{~Gy}$ in 30 fractions. The radiation treatment volume included the metastatic liver mass alone with an adequate margin. During radiotherapy, the patient's symptoms rapidly disappeared and his liver function rapidly normalized (Table 1). During the latter part of the

Table 1. Changes in the liver function during and after radiotherapy (unit, IU/L)

\begin{tabular}{cccccc}
\hline $\begin{array}{c}\text { Measured } \\
\text { date }\end{array}$ & AST & ALT & LDH & ALP & GGT \\
\hline $08 / 31 / 2010$ & 63 & 48 & 1,152 & 278 & 282 \\
$10 / 20 / 2010$ & 29 & 23 & 660 & 131 & 115 \\
$10 / 26 / 2010$ & 29 & 27 & 489 & 110 & 105 \\
$11 / 01 / 2010$ & 28 & 30 & 412 & 112 & 102 \\
$11 / 08 / 2010$ & 32 & 43 & 373 & 103 & 100 \\
$11 / 16 / 2010$ & 32 & 41 & 362 & 94 & 98 \\
$11 / 23 / 2010$ & 36 & 38 & 381 & 104 & 95 \\
$11 / 29 / 2010$ & 45 & 43 & 402 & 121 & 95 \\
$05 / 24 / 2012$ & 22 & 19 & - & 96 & - \\
$07 / 30 / 2014$ & 16 & 14 & 177 & 79 & 44 \\
\hline
\end{tabular}

AST, aspartate transaminase; ALT, alanine transaminase; LDH, lactic acid dehydrogenase; ALP, alkaline phosphatase; GGT, gammaglutamyl transpeptidase. radiotherapy, the metastatic mass was markedly diminished on the FDG PET-CT scan (Fig. 1D). His CEA level, which was 1,736 $\mathrm{ng} / \mathrm{mL} \mathrm{ng}$ before radiotherapy, abruptly decreased to $6.9 \mathrm{ng} /$ $\mathrm{mL}$ in March 2011 and to $3.2 \mathrm{ng} / \mathrm{mL}$ by December 2012. After about four years from the first radiotherapy, the patient's most recent CEA level (July 30, 2014) was $3.5 \mathrm{ng} / \mathrm{mL}$ (Fig. 2). After nearly four years since the first radiotherapy treatment, the patient has had consistent perfect normal liver function, which was confirmed in recent laboratory studies on July 30, 2014

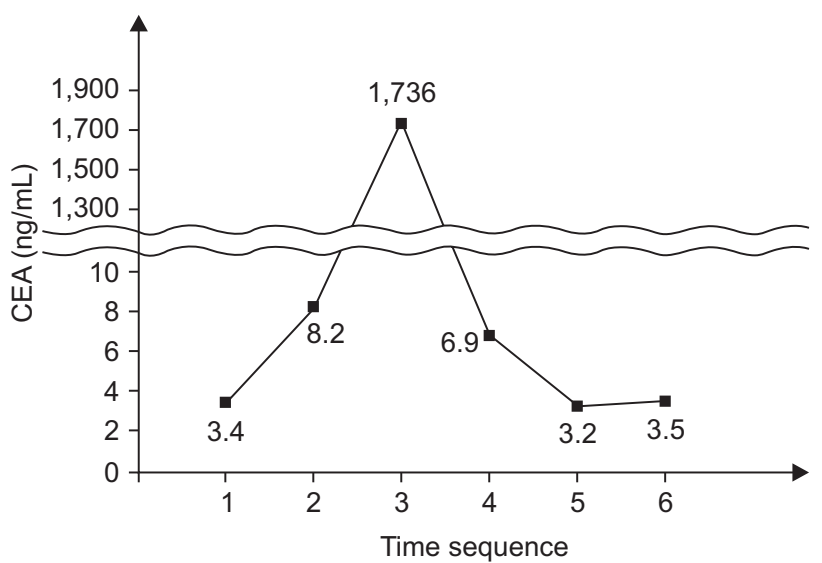

Fig. 2. Carcinoembryonic antigen (CEA) level rapidly decreased after radiotherapy for liver metastasis. Time sequences 1, after curative resection of primary colon cancer; 2 , at first detection of liver metastasis (September 2008); 3, immediately before liver radiotherapy (September 2010); 4, three months after liver radiotherapy (March 2011); 5, one and half years after liver radiotherapy (December 2012); 6, in recent time (July 2014).


Fig. 3. First lung metastasis completely regressed with radiotherapy. Arrows denote first lung metastasis. (A) Pre-radiotherapy ${ }^{18} \mathrm{~F}$-fluorodeoxyglucose (FDG) positron emission tomography/computed tomography (PET/CT) scan showed a hypermetabolic lesion in right upper lobe (RUL) of lung. (B) Pre-radiotherapy chest CT scan showed a 2-cm size metastatic mass in RUL of lung. (C) In postradiotherapy FDG PET/CT scan, no abnormal hypermetabolic lesion was observed. 
(Table 1).

After the completion of the radiotherapy for his liver metastasis, we strongly recommended chemotherapy again, but the patient did not received further chemotherapy after receiving one cycle of fluorouracil-leucovorin chemotherapy in January 2011. He did not undergo any further chemotherapy.

In March 2013, a whole body FDG PET-CT scan showed a hot uptake, suggesting lung metastasis, was found in the medial portion of the patient's right upper lobe (RUL) of lung (Fig. $3 \mathrm{~A}$ ). The previously treated liver metastasis was in a complete state of remission in the same FDG PET-CT scan (Fig. 3A). Chest CT scan showed a 2-cm size metastatic mass in RUL of lung (Fig. 3B). As a result, the patient underwent a second course of radiotherapy of $70 \mathrm{~Gy} / 35$ fractions for his lung metastasis from April 25, 2013 to July 3, 2013. After the second radiation treatment, no residual mass was found on his right lung and there was no evidence of recurred malignancy in the other site on a subsequent FDG PET-CT scan (Fig. 3C).

During an additional follow-up appointment, a $10 \mathrm{~mm}$-sized nodule was found in RUL anterolateral subpleural portion of lung on CT scan at September 3, 2013, suggesting metastasis. The mass size increased to $13 \mathrm{~mm}$ on a subsequent CT scan on November 11, 2013 (Fig. 4A). So, a third course of radiotherapy including $75 \mathrm{~Gy} / 30$ fractions was performed on his new lung nodule from November 25, 2013 to January 22, 2014. Three months after the third round of radiotherapy, the treated nodule showed a complete response on the CT scan (Fig. 4B), and there were no abnormal hypermetabolic lesions in other sites on the FDG PET-CT scan.

He is now, nearly four years from his first radiation treatment for liver metastasis, and after three subsequent curative dose radiation treatments, fully active with an Eastern Cooperative Oncology Group (ECOG) scale of 0 and without any evidence of cancer.

Also of note, the patient ate a continuous diet of Fucoidan products throughout his battle with cancer.

\section{Discussion}

There were sequential changes in the concept of cancer progression.

Traditionally, the Halsted [11] model, which describes a cancer spread in an orderly, extending, contiguous fashion, from the primary tumor through the lymphatic system to the lymph nodes, and then to distant metastatic sites, has been widely accepted.

A subsequent hypothesis suggested that cancer is a systemic disease, and implied that small tumors are just an early manifestation of the systemic disease, which, if it is to metastasize, has already metastasized, and nodal involvement is not an orderly contiguous extension, but rather a marker of the distant disease. The systemic disease hypothesis was fully explicated with both laboratory and clinical studies by Fisher [12].

A third, 'Hellman and Weichselbaum' [1] theory of spectrum hypothesis suggests a multistep cancer progression that
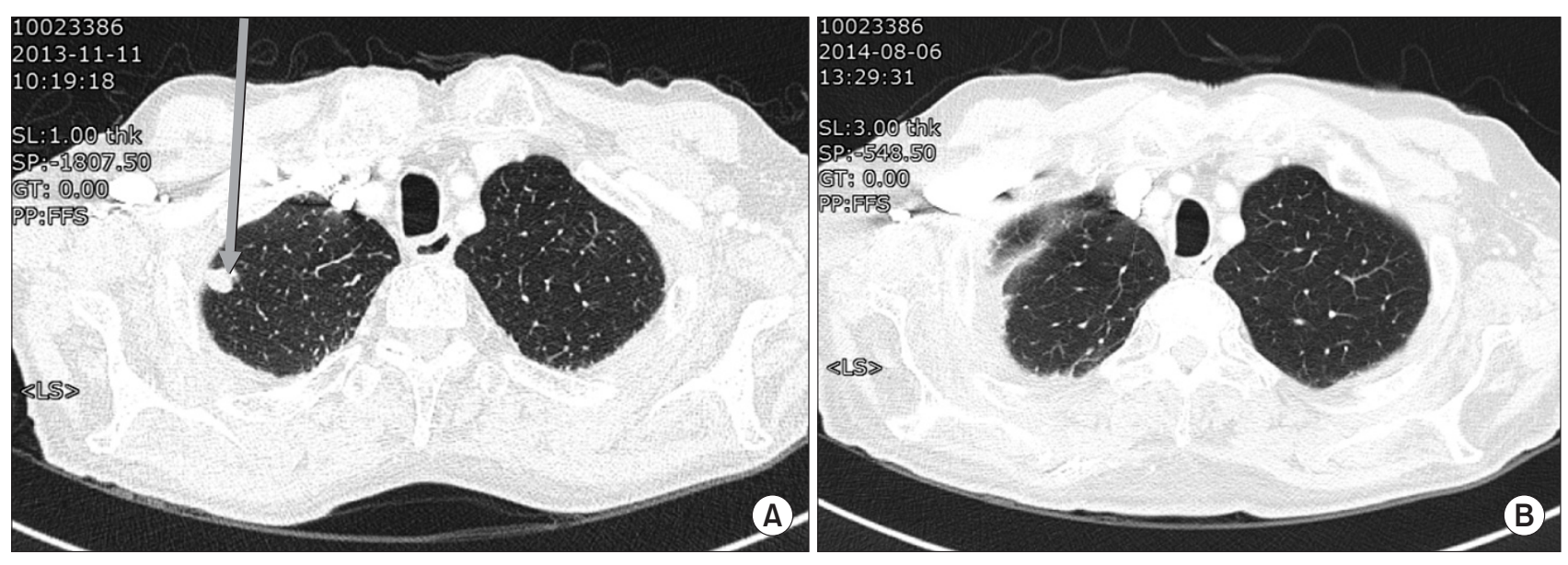

Fig. 4. Second lung metastasis completely regressed with radiotherapy. Arrows denote second lung metastasis. (A) Pre-radiotherapy chest computed tomography (CT) scan showed increased size of new right upper lobe (RUL) nodule compared with previous CT scan $(10 \rightarrow 13 \mathrm{~mm}$ ) suggesting aggravated single hematogenous lung metastasis. (B) In post-radiotherapy chest CT scan, a nodule in RUL of lung disappeared and subpleural consolidation with focal ill-defined ground-glass opacity suggesting radiation change appeared in posterolateral portion of RUL. 


\section{Hyong Geun Yun}

describes a cancer ranging between a disease that remains localized and disease that is systemic at the time of diagnosis.

So, the implication is that oligometastatic disease is an intermediate state of cancer spread, between the localized disease and widespread metastases, and therefore, may be cured with metastasis-directed therapy.

Oligometastases, the state in which the patient shows distant relapses in only a limited number of regions, could be described as an uncontrolled primary site with several distant metastases. Niibe and Hayakawa [2] and Niibe et al. [3-6] proposed the new notion of oligorecurrence to overcome these descriptive problems. Oligorecurrence is a notion similar to oligometastases. However, the conditions of oligorecurrence are (i) one to several distant metastases/recurrences (usually one) in one to several organs (usually one); (ii) primary site of the cancer controlled; (iii) one to several distant metastases/ recurrences can be treated with local therapy; and (iv) no other distant metastases/recurrences other than those in (iii).

Niebe et al. [6] also proposed the Niibe-Onishi-Chang classification as a more detailed classification of oligometastases and oligorecurrence. This classification includes not only oligometastases, but also poly-metastases. Oligometastases and oligorecurrences usually offer a better prognosis than polymetastases. However, oligometastases and oligorecurrence are cancer-specific and organ-specific. The appearance status of oligometastases or oligorecurrence is thus sometimes equivalent to poly-metastases, such as in pancreatic cancer, sarcoma, or malignant melanoma. One or two, lung or liver metastases of colon or rectal cancer (same with our case) are classified as favorable prognostic group [6].

The improvement of systemic chemotherapy, including molecular-targeted therapy, has allowed micro-metastases to be almost completely eradicated clinically. Theoretically, if several gross metastatic or recurrent sites could be eradicated by local therapy, these patients could be cured with concomitant systemic chemotherapy $[2,13]$. So, in the treatment of patients with oligometastases, systemic treatment is strongly recommended in addition to local radiation.

But for elderly patients, age-related biological changes are associated with an increased susceptibility to toxicity during chemotherapy [7]. Additionally, older patients and their family members may be more reluctant to select chemotherapy than younger patients. On the other hand, radiation is generally safe and well-tolerated, as 75\%-90\% of older patients complete the therapy. New modalities of radiation, including intensity-modulated radiotherapy, tomotherapy [14], GammaKnife, 3-dimensional conformal therapy, or brachytherapy can reduce toxicity via organ sparing and limiting the total number of required treatment sessions.

In addition, some tumors in the elderly tend to be less aggressive. In some tumors types, such as breast cancer [8] and non-small cell lung cancer [9], a better prognosis with increased age has been suggested. On the contrary, in some tumor types, such as diffuse large cell lymphoma [15], a worse prognosis with increased age has been suggested.

On the other hand, in a large Netherlands study, despite of the more frequent comorbidity and insufficient treatment in elderly patients, the relative survival was not lower for colon cancer, prostate cancer, or indolent non-Hodgkin lymphoma $(\mathrm{NHL})$ than in younger patients [10]. We think these results suggest that colon cancer, breast cancer, non-small cell lung cancer, and indolent NHL may show a better prognosis with age.

According to a recent large epidemiologic study, the incidence and mortality of cancer usually decreases or plateaus at very old ages. The prevalence of various cancers usually decreases swiftly in people over the age of 90 . When there was statistical power, the incidence normally peaked between ages 75 years and 90 years, dropping off abruptly afterward and trending toward zero among centenarians [16].

As described in this case report, our elderly patient with repeated oligorecurrence strongly refused chemotherapy. Consequently, in order to treat oligorecurrence without chemotherapy, we thought curative doses of radiation therapy may be helpful, especially in very old patients.

In our 87-year-old patient, single oligorecurrences occurred repeatedly three times in the liver and lung during a six-year period, and all the oligorecurrences were cured with curative doses of radiotherapy alone. Currently, nearly four years after his first radiation treatment for liver metastasis, the patient is alive, has a very good score on the ECOG scale (0), and has no evidence of any cancer.

\section{Conflict of Interest}

No potential conflict of interest relevant to this article was reported.

\section{References}

1. Hellman $\mathrm{S}$, Weichselbaum RR. Oligometastases. J Clin Oncol 1995;13:8-10.

2. Niibe $Y$, Hayakawa K. Oligometastases and oligo-recurrence: the new era of cancer therapy. Jpn J Clin Oncol 2010;40:107- 
11.

3. Niibe $Y$, Kazumoto $T$, Toita $T$, et al. Frequency and characteristics of isolated para-aortic lymph node recurrence in patients with uterine cervical carcinoma in Japan: a multi-institutional study. Gynecol Oncol 2006;103:435-8.

4. Niibe $Y$, Kenjo M, Kazumoto $T$, et al. Multi-institutional study of radiation therapy for isolated para-aortic lymph node recurrence in uterine cervical carcinoma: 84 subjects of a population of more than 5,000. Int J Radiat Oncol Biol Phys 2006;66:1366-9.

5. Niibe $Y$, Kuranami M, Matsunaga $K$, et al. Value of highdose radiation therapy for isolated osseous metastasis in breast cancer in terms of oligo-recurrence. Anticancer Res 2008;28:3929-31.

6. Niibe $Y$, Chang JY, Onishi H, Salama J, Hiraki T, Yamashita H. Oligometastases/oligo-recurrence of lung cancer. Pulm Med 2013;2013:438236.

7. Stec R, Bodnar L, Smoter M, Maczewski M, Szczylik C. Metastatic colorectal cancer in the elderly: an overview of the systemic treatment modalities (review). Oncol Lett 2011;2:311.

8. Petrakis IE, Paraskakis S. Breast cancer in the elderly. Arch Gerontol Geriatr 2010;50:179-84.
9. Repetto L, Balducci L. A case for geriatric oncology. Lancet Oncol 2002;3:289-97.

10. Janssen-Heijnen ML, Houterman S, Lemmens VE, Louwman MW, Maas HA, Coebergh JW. Prognostic impact of increasing age and co-morbidity in cancer patients: a population-based approach. Crit Rev Oncol Hematol 2005;55:231-40.

11. Halsted WS. The results of operations for the cure of cancer of the breast performed at the Johns Hopkins Hospital from June, 1889, to January, 1894. Ann Surg 1894;20:497-555.

12. Fisher B. Laboratory and clinical research in breast cancer: a personal adventure: the David A. Karnofsky memorial lecture. Cancer Res 1980;40:3863-74.

13. Punglia RS, Morrow M, Winer EP, Harris JR. Local therapy and survival in breast cancer. N Engl J Med 2007;356:2399-405.

14. Choi Y, Kim JW, Lee IJ, Han HJ, Baek J, Seong J. Helical tomotherapy for spine oligometastases from gastrointestinal malignancies. Radiat Oncol J 2011;29:219-27.

15. Klapper W, Kreuz M, Kohler CW, et al. Patient age at diagnosis is associated with the molecular characteristics of diffuse large B-cell lymphoma. Blood 2012;119:1882-7.

16. Harding C, Pompei F, Wilson R. Peak and decline in cancer incidence, mortality, and prevalence at old ages. Cancer 2012;118:1371-86. 